\title{
Development of cement-based mortars for 3D printing through wet extrusion
}

\author{
Behzad Zahabizadeh, Vítor M.C.F. Cunha \\ ISISE, Institute of Science and Innovation for Bio-Sustainability (IB-S), Department of Civil \\ Engineering, University of Minho, Guimarães, Portugal.
}

\section{João Pereira}

Innovation Point, Rua de Pitancinhos - Palmeira, 4700-727, Braga, Portugal.

\section{Claúdia Gonçalves}

DST group, Rua de Pitancinhos - Palmeira, 4700-727, Braga, Portugal.

\begin{abstract}
The construction sector is connoted as an extremely traditional business sector since long ago. However, due to the increase of the global competiveness, there is a demand on the development of new building materials and construction methods that can bring added value to the companies. The 3D concrete printing is a novel construction approach within digital construction that can offer a higher degree of optimization and flexibility for producing either structures or structural elements with complex geometries. One of the main challenges in the 3D concrete printing using wet extrusion is balancing properly the rheological and mechanical properties of the printable mixtures. In this study, several mixtures were developed and their capability for being used in 3D printing was assessed and discussed based on their rheological properties. The compressive strength of the matrices that could be properly printed are also presented.
\end{abstract}

Keywords: Digital construction; 3D concrete printing; Wet extrusion; Rheological properties.

\section{Introduction}

Additive manufacturing or solid free-form construction based on a layered fabrication process is one of the novel approaches based on the application of digitized technologies that are being introduced in the construction industry [1,2]. Although the growth rate of embodying digital technologies within the Architecture, Engineering and Construction (AEC) sector has not been the same as other more emergent fields, such as aerospace or automotive industry [2], nowadays the digital construction is one of the most promising revolutionary approaches within the construction industry. The main idea of digital construction is solving the existing difficulties in the conventional methods of construction $[2,3]$. In this case, the $3 D$ concrete printing (3DCP), which is based on additive manufacturing (AM) technique can offer a higher degree of optimization and 
flexibility for producing either structures or structural elements with complex geometries.

The ability of producing more complex architectural geometries without increasing the construction costs (i.e. the geometry complexity is obviated from the production cost), the addition of multi-functionality and / or functional graded properties to both architectural and structural elements produced with this technique are some benefits that $3 D C P$ can bring to the construction industry [1,3-8].

Contour crafting $(\mathrm{CC})$, concrete printing $(\mathrm{CP})$, and $D$-shape are three of the most well-known methods for 3DCP. CC and CP are based on wet extrusion $[10,11]$, while $D$-shape is a powder-based method [12]. Generally, in the wet extrusion process, the material development for the 3D printing process is based on the optimization of the material's fresh-state behaviour and its compatibility with the employed 3D printing technology [13]. Control of rheological behaviour of the mixture during the printing process is a vital task in order to ensure an uniform flow of material during extrusion, as well as a suitable setting of mixture afterwards [14]. Therefore, extrudability, buildability, and open time are critical material properties within the fresh state that should be complied in order to design suitable mixture compositions for 3DCP.

The mixtures for 3D printing must show the ability of being uniformly extruded through the nozzle for each layer while this process is continued up until the entire element is built layer by layer. Moreover, during the printing process, the extruded mixture (i.e. printed object) must not exhibit any kind of collapse or excessive deformations caused by the material's self-weight [15]. The open time is another critical feature for printable mixtures, which can affect both extrudability and buildability [15] and is controlled by the dynamic viscosity of mixture [14]. Therefore, one of the main challenges regarding using $3 D C P$ is related to properly balance the rheological properties and the mechanical properties of printable compositions. Achieving this balance is of uttermost importance and mainly depends on several issues such as the type of material, method of printing, desired geometry of printed specimen, and predefined parameters for the printer machine [15-19].

2. As previously discussed, 3D printing technology can revolutionize the prevailing traditional construction methods within the AEC sector, thus propelling the development of innovative structural solutions with extremely complex geometries and concomitantly obviate the increase of cost due to complexity. One of the main challenges is the development of suitable cementitious matrices that could be used with these novel techniques, thus assuring good rheological properties for a proper quality control of the printed elements. In this work, it was assessed the printing capability based on a wet extrusion technique of distinct matrices and the influence of its fresh state properties on the quality of the printed specimens. Finally, the compressive strength of the matrices that could be properly printed is also presented. Materials and mixture selection

In this study, some empirical rheological tests on five distinct compositions (see Table 1) were carried out in order to select mortar mixtures with suitable rheological properties for 3D printing. The selection of the mixture was done based on the best compatibility between the rheological properties of the mortar (i.e. in the fresh state) and the printing technology.

The main required rheological properties of a mortar mixture to be used in 3D printing include 
extrudability and buildability, which are considerably affected by the workability and open time. The printing speed, flow rate, geometrical dimensions of layers, and time gap between the layers all are some of the required parameters that must also be set accurately based on the measured rheological properties of the mortar mixture.

In this study, five cement-based mortar mixtures, namely Mix A to Mix D with a cement content lower than $300 \mathrm{~kg} / \mathrm{m}^{3}$ and Mix E with less than $450 \mathrm{~kg} / \mathrm{m}^{3}$ of cement were prepared to compare their rheological behaviour and select the best one for the designed 3D printer. All compositions were defined based on a fine river sand with a maximum size of $1 \mathrm{~mm}$. A maximum size of $1 \mathrm{~mm}$ sand was selected because of the limitation of smallest dimension of the nozzle (i.e. $12 \mathrm{~mm}$ ) and due to better surface finishing. The binder comprised cement CEM I $42.5 \mathrm{R}$, fly ash, and silica fume, which after some preliminary tests were kept constant for Mix A to Mix D. However, in Mix E the binder components were adjusted to have into account the increase of cement content. Furthermore, the water to binder ratio (W/B) was also kept constant for all mixtures.

Table 1. Mortar mixture compositions $\left(\mathrm{kg} / \mathrm{m}^{3}\right)$

\begin{tabular}{lccccc}
\hline \multirow{2}{*}{ Material } & \multicolumn{5}{c}{ Mixture compositions $\left(\mathrm{kg} / \mathrm{m}^{3}\right)$} \\
\cline { 2 - 6 } & Mix A & Mix B & Mix C & Mix D & Mix E \\
\hline sand & 1183 & 1183 & 1183 & 1183 & 1201 \\
\hline Cement & 286 & 286 & 286 & 286 & 436 \\
\hline Fly ash & 423 & 423 & 423 & 423 & 285 \\
\hline Silica fume & 79 & 79 & 79 & 79 & 20 \\
\hline Water & 248 & 248 & 248 & 248 & 252 \\
\hline Superplasticizer & $8.9(1 \%)$ & $10.2(1.3 \%)$ & $11.8(1.5 \%)$ & $15.8(2 \%)$ & $10.4(1.3 \%)$ \\
\hline
\end{tabular}

Finally, a polycarboxylate based superplasticizer was selected to control the workability, open time and hence the extrudability and buildability of the mortar mixtures. The superplasticizer content used was $1,1.3,1.5$ and $2 \%$ by weight of the total binder for Mix A to Mix D, respectively. Based on the assessment of the fresh state behaviour of Mix A to D, Mix $E$ was prepared with $1.3 \%$ of superplasticizer.

\section{Concrete printing technology}

A small 3D printer developed from scratch with a printable volume of nearby $0.4 \times 0.4 \times 0.4 \mathrm{~m}$ was used in this study. The printer is comprised of an aluminium frame as a support for a stainless steel funnel with an auger inside. All the movements of printing systems are controlled by stepper motors along $X, Y$, and $Z$ directions, as well as for the rotation of the auger inside the funnel. Figure (1) depicts a general view of the 3D printer.

Before starting the extrusion procedure for printing the specimens, the fresh mortar mixture is placed into funnel. Then, the printing process will start based on predefined parameters for the 3D printer machine and rheological behaviour of the mortar. These parameters include: i) speed of printing, ii) flow rate, and iii) geometrical dimensions of the specimens / layers.

During the printing process, the fresh material moves smoothly inside the funnel using the rotation auger under its own self-weight. Finally, the material passes through a customized nozzle to build layer upon layer the desired geometry of the specimens. 


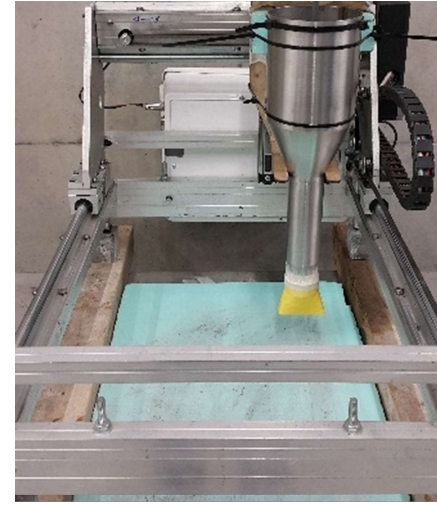

Figure 1. General view of 3D printer

\section{Fresh properties evaluation}

\subsection{Workability}

The flow table test [20] was used as a simple method for measuring the workability of mortar mixtures. Although this conventional test method cannot measure the physical properties of the material in fundamental units, it can demonstrate some qualitative data regarding the proper workability required for the 3D printing process.

In each flow test the workability of the mixtures was measured in two steps: i) measuring the workability after removing the mould and before jolting the flow table, ii) measuring the workability after 15 times shaking the flow table.

This two-steps can roughly simulate the situation of material during 3D printing process. During the printing, the material flows under its own selfweight using the rotation of auger (similar to shaking the flow table; i.e. step ii). After extruding, the material must keep stationary at its final position without exhibiting deformation (similar to situation of removing the mould and before shaking the flow table, i.e. step i). The average workability for Mix A to Mix E at step (i) was 100, $115,130,130$, and $115 \mathrm{~mm}$, respectively. At the step (ii), the average workability was equal to 173 , 193, 208, 210, and $193 \mathrm{~mm}$ for Mix A to Mix E, respectively.

\subsection{Printability}

Printability is the ability of the material flowing uniformly inside the extruder and during the extrusion process itself when printing the layers. The printability was evaluated based on the maximum possible printable distance of a layer with $60 \mathrm{~mm}$ wide filament extruded from a nozzle with the size of $60 \times 12 \mathrm{~mm}$.

Mix A with $1 \%$ superplasticizer was too stiff even instantly after the end of the mixing process. During the extrusion process, cracks and discontinuities in the printed layers were quite visible. On the other hand, Mix B with $1.3 \%$ of superplasticizer exhibited a uniform flow when filling the printer's container, as well as during the extrusion from the nozzle without showing any matrix separation. The printed layers using Mix B were placed accurately in their location and at the surface no cracks were visible. Finally, Mix $C$ and Mix D with 1.5 and 2\% of superplasticizer, respectively, were too flowable after mixing. Moreover, right after the extrusion, due to a low shear yield stress of both Mix $C$ and $D$, the layers expanded transversely even before supporting the weight of the upper layer.

Regarding printability of the mixtures with lower cement content, Mix B with $1.3 \%$ of superplasticizer was the one that exhibited better properties. For Mix E based on a higher cement content, i.e. $436 \mathrm{~kg} / \mathrm{m}^{3}$, the same amount of superplasticizer and water to binder ratio of Mix $B$ was used. This mixture also showed a good printing quality without any crack or matrix separation during the extrusion procedure. Figure (2) shows an overview of the printed layers' quality by using Mix A to Mix E, respectively.

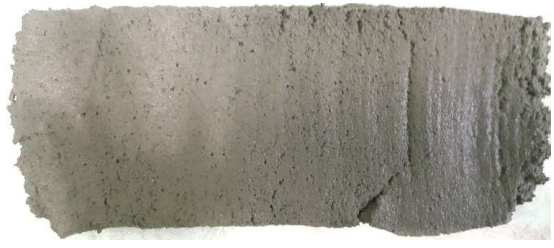

(a)

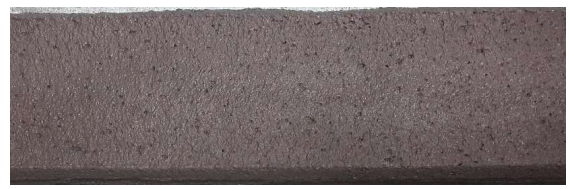

(b) 


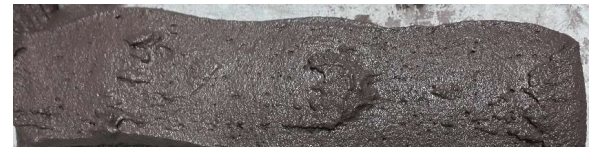

(c)

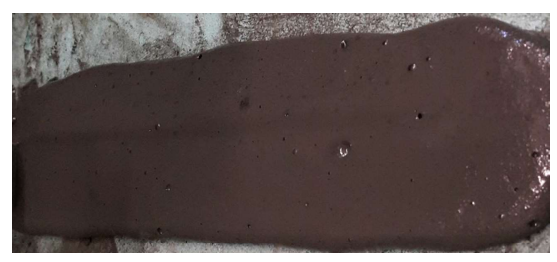

(d)

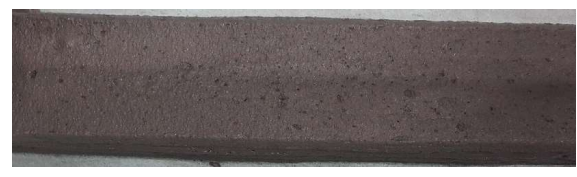

(e)

Figure 2.Printability of (a) Mix A, (b) Mix B, (c) Mix $C$, (d) Mix D, and (e) Mix E

Considering the influence of workability on the printability of the tested mixtures, it was concluded that an average flow diameter of 115 and $193 \mathrm{~mm}$ before and after shaking the flow table, respectively, were the optimal values for having a good continuous and homogeneous printing.

The aforementioned average flow values were obtained for Mix B and Mix E, which have shown the best printability between all the mixtures. It should be noted that this conclusion is also related to the 3D printer system and the predefined parameters such as flow rate, printing speed, and geometrical dimensions of layers / specimens.

\subsection{Buildability}

Buildability can be regarded as the ability of the material after being extruded to retain its initial dimensions and shape, under loads. Furthermore, each printed layer must have enough strength to support its own self-weight and also the weight of the top layers without collapsing and even without showing deformation. Additionally, during the printing, a good interface bond between the layers should be attained. Therefore, at a first instance, the buildability was evaluated based on the maximum number of consecutive layers that could be stacked on top of each other. Note that, this assessment was performed for a mixture without accelerator and in the beginning of the open time interval (i.e. just after mixing). Then, the size and shape of the printed layers was visually assessed.

Even though Mix B and Mix E were selected as the best mixtures regarding their printability, in addition the buildability of other compositions was also investigated in order to compare the results. The maximum number of printable layers of the mixtures that exhibited the best printability capability, i.e. Mix B and Mix E, was 9 layers, which was equivalent to $90 \mathrm{~mm}$ height (based on the specified layer height of $10 \mathrm{~mm}$ ). This buildability was accessible immediately after preparation of the mixture, thus more to the end of the open time interval a higher number of layers would be obtained. The final printed specimen have shown that the layers were able to maintain their size and shape, Figures (3b) and (3e). Mix A was too stiff right after mixing, thus it was possible to print 14 layers on top of each other, however without a proper printability, since cracks and separation of the matrix was also visible, Figure (3a). For Mix C (1.5\% of superplasticizer) it was possible to print 5 layers, Figure (3c). However, in Mix C, its viscosity was increased due to the higher ratio of superplasticizer, thus there was some difficulty regarding the layer positioning and surface finishing due to stickiness of the mixture to the extruder nozzle. Finally, Mix D (with 2\% of superplasticizer) was too flowable still after about more than one and half hour and so only two layers were printable using that. Moreover, these two layers almost merged to each other due to the lack of plastic yield strength, Figure (3d).

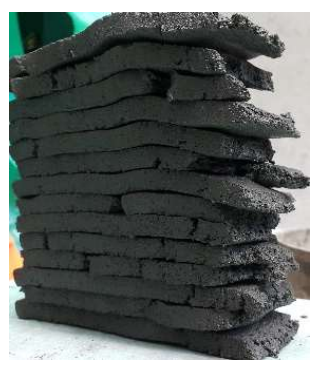

(a) 


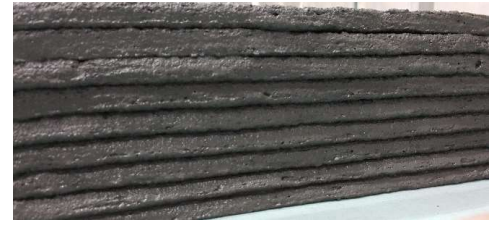

(b)

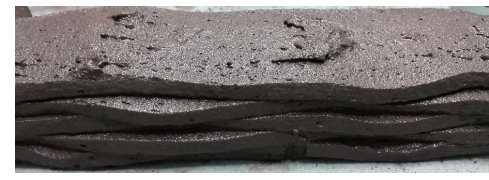

(c)

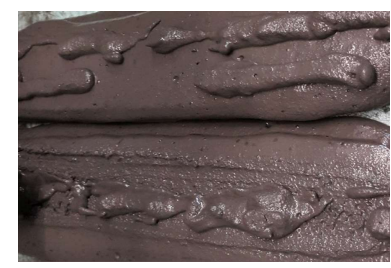

(d)

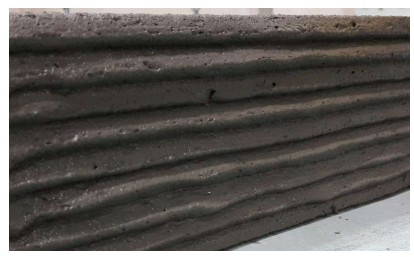

(e)

Figure 3. Buildability of (a) Mix A, (b) Mix B, (c) Mix C, (d) Mix D, and (e) Mix E

\subsection{Open time}

Open time is another critical property of a printable material. This property can affect both printability and buildability of mixture during time. Long open time is needed for keeping a stable flow during the printing process. On the other hand, a long open time can have reverse effect on the buildability through the possible excessive deformation of layers if no accelerator is used immediately before or after extruding the layers. Therefore, finding the optimum duration of open time for each mixture based on the printer definitions and also the geometrical dimensions of the desired object is important.

In this study, the open time was measured based on a continuous printing of a mixture until the final time that the mixture was still printable uniformly and buildable without any blockage in the extruder's nozzle.

For Mix A ( $1 \%$ of superplasticizer), less than 1 hour of open time was obtained. On the other hand, although using 1.5 and $2 \%$ of superplasticizer has increased the setting time of Mix C and Mix D, respectively, it was not possible to measure the real open time. Because the real open time is regarded as the time that the material is printable and also buildable with proper quality. Hence, Mix $C$ and Mix D were printable and buildable only after around 1.3 hours from mixture preparation, however with the lack of required plastic yield strength to maintain size and dimensions.

Finally, for Mix B and Mix E (both with 1.3\% of superplasticizer), the open time was around 2 and 1 hours, respectively. Both mixtures were printable and buildable instantly after mixture preparation until the end of open time. The difference of open time for these two mixtures using the same amount of superplasticizer can be ascribed to the different cement contents. The higher cement content for Mix E (i.e. $436 \mathrm{~kg} / \mathrm{m}^{3}$ ) than the one in Mix B (i.e. $286 \mathrm{~kg} / \mathrm{m}^{3}$ ) lead to the acceleration of cement hydration process in Mix E and so resulted in the less open time.

\section{Mechanical characterization}

The assessment of the compressive strength $\left(f_{\mathrm{cm}}\right)$ of the studied mixtures was done exclusively on the selected mixtures, namely, Mix B and Mix E, which was based on their printing performance.

Two slabs with the size of $300 \times 60 \times 50 \mathrm{~mm}$ and $200 \times 60 \times 50 \mathrm{~mm}$ were printed using both Mix B and Mix E. After nearby 28 days of curing, the required specimens for compressive strength tests were cut and rectified from the printed slabs. The specimen dimensions were $40 \times 40 \times 80 \mathrm{~mm}$. Unlike mouldcast samples, the printed specimens may show an anisotropic behaviour due to the influence of the loading direction regarding the printed layer's orientation. The compressive behaviour of the printed specimens was assessed based solely on the application of the load parallel to the layer's direction, since the main scope of this paper was to design matrices and assess their requirements in 
the fresh state to be used in 3D printing of concrete. A detailed discussion about the influence of loading direction regarding the printing direction on the compressive behaviour of printed specimens can be found elsewhere [21]. For each series, five printed specimens and five mould cast specimens were used.

The average $f_{\mathrm{cm}}$ at 28 days for Mix B was $51.0 \mathrm{MPa}$ $(\mathrm{CoV}=7.86 \%)$ and $58.0 \mathrm{MPa}(\mathrm{CoV}=2.98 \%)$ for the printed specimens and mould cast series, respectively. On the other hand, Mix E at 28 days shown the average $f_{\mathrm{cm}}$ of $70.1 \mathrm{MPa}(\mathrm{CoV}=1.62 \%$ ) and $75.6 \mathrm{MPa}(\mathrm{CoV}=3.25 \%)$ for series that were printed and mould cast, respectively.

Mix $E$ had a higher compressive strength when compared to Mix B, as it was expected due to a higher amount of cement. Moreover, the printed series in both mixtures had a lower compressive strength than the mould-cast series, in particular for Mix B this reduction was $12 \%$. On the other hand, for Mix E, the compressive strength decrease was only about $7 \%$. For this technology, these differences are quite acceptable, since proper rheological properties of the mixtures guaranteed a good quality of printing in this study.

\section{Conclusions}

In this study the required fresh state behaviour for selecting a suitable mortar mixture to be used in 3D concrete printing was investigated. The main characteristics in the fresh state of these kind of mixtures include printability and buildability, which are affected by the open time and workability.

Five cement-based mortar mixtures were prepared based on the same amount of water to binder ratio $(W / B=0.31)$. Mix $A$ to Mix $D$ prepared with a cement content of $286 \mathrm{~kg} / \mathrm{m}^{3}$ and $1,1.3,1.5$ and $2 \%$ of superplasticizer (by wt. of binder), respectively. The results have shown that Mix B with $1.3 \%$ of superplasticizer had the best printability and buildability. Using this mix, it was possible to print a layer with maximum length of $322 \mathrm{~mm}$ and the height of $90 \mathrm{~mm}$ (9 layers of $10 \mathrm{~mm}$ ). The open time of Mix B was approximately 2 hours.

Moreover, a mixture with higher cement content $\left(436 \mathrm{~kg} / \mathrm{m}^{3}\right)$ was prepared based on the optimal superplasticizer ratio found for Mix B. Mix E also has shown good printability and buildability. However, due to the higher cement content, thus faster hydration process, the effective open time of Mix E decreased from 2 hours to around 1 hour.

The compressive strength test revealed that there was decrease of 12 and $7 \%$ on $f_{\mathrm{cm}}$ for the printed mixtures, i.e. Mix $B$ and Mix $E$, when compared to the mould cast ones, respectively.

\section{References}

[1] Khoshnevis B. Automated construction by contour crafting-related robotics and information technologies. Automation in Construction 2004; 13: 5-19.

[2] Buswell RA, Soar RC, Gibb AGF, et al. Freeform Construction: Mega-scale Rapid Manufacturing for construction. Automation in Construction 2007; 16: 224-231.

[3] Lim S, Buswell RA, Le TT, et al. Developments in construction-scale additive manufacturing processes. Automation in Construction 2012; 21: 262-268.

[4] Perrot A, Rangeard D, Pierre A. Structural built-up of cement-based materials used for 3D-printing extrusion techniques. Materials and Structures 2016; 49: 1213-1220.

[5] Buswell RA, Soar RC, Pendlebury M, et al. Investigation of the potential for applying freeform processes to construction. In: IN: Proceedings of the 3rd International Conference on Innovation in Architecture, Engineering and Construction (AEC). Rotterdam, Netherlands, pp. 141-150.

[6] Hopkinson N, Dicknes P. Analysis of rapid manufacturing-using layer manufacturing processes for production. Proceedings of the Institution of Mechanical Engineers, Part C: Journal of Mechanical Engineering Science 2003; 217: 31-39.

[7] Hopkinson N, Gao Y, McAfee DJ. Design for environment analyses applied to rapid manufacturing. Proceedings of the Institution of Mechanical Engineers, Part D: Journal of 
Automobile Engineering 2006; 220: 13631372.

[8] Lim S, Buswell R, Le T, et al. Development of a viable concrete printing process. In: 28th International Symposium on Automation and Robotics in Construction (ISARC2011). Seoul, South Korea, pp. 665-670.

[9] Feng P, Meng X, Chen J-F, et al. Mechanical properties of structures 3D printed with cementitious powders. Construction and Building Materials 2015a; 93: 486-497.

[10] Khoshnevis B, Bukkapatnam S, Kwon H, et al. Experimental investigation of contour crafting using ceramics materials. Rapid Prototyping Journal 2001; 7: 32-42.

[11] Lim S, Le T, Webster J, et al. Fabricating construction components using layer manufacturing technology. In: Global Innovation in Construction Conference 2009 (GICC'09). Loughborough University, 2009.

[12] Cesaretti G, Dini E, De Kestelier X, et al. Building components for an outpost on the Lunar soil by means of a novel 3D printing technology. Acta Astronautica 2014; 93: 430450.

[13] Ma G, Wang L. A critical review of preparation design and workability measurement of concrete material for largescale 3D printing. Frontiers of Structural and Civil Engineering 2017; 12: 382-400.

[14] Weng Y, Lu B, Tan MJ, et al. Rheology and Printability of Engineered Cementitious Composites-A Literature Review. In: Proceedings of the 2nd International Conference on Progress in Additive Manufacturing (Pro-AM 2016). 2016.

[15] Le TT, Austin SA, Lim S, et al. Mix design and fresh properties for high-performance printing concrete. Materials and Structures 2012a; 45: 1221-1232.
[16] Le TT, Austin SA, Lim S, et al. Hardened properties of high-performance printing concrete. Cement and Concrete Research 2012b; 42: 558-566.

[17] Gosselin C, Duballet R, Roux P, et al. Largescale 3D printing of ultra-high performance concrete - a new processing route for architects and builders. Materials \& Design 2016; 100: 102-109.

[18] Malaeb Z, Hachem H, Tourbah A, et al. 3D concrete printing: machine and mix design. IJCIET 2015; 6: 14-22.

[19] Nerella VN, Krause $M$, Näther $M$, et al. Studying printability of fresh concrete for formwork free Concrete on-site 3D Printing technology (CONPrint3D). In: 25th Confernce on Rheology of Building Materials. Regensburg, Germany, 2016.

[20] British Standards Institution. BS EN 10153:1999. Methods of test for mortar for masonry. Determination of consistence of fresh mortar (by flow table).

[21] Zahabizadeh B, Pereira J, Gonçalves C, et al. The effect of loading direction on the compressive behaviour of a 3D printed cement-based material. In: IABSE Symposium 2019 Guimarães: Towards a Resilient Built Environment - Risk and Asset Management. Guimarães, Portugal, 2019. 